\title{
Collision Tumor Composed of an Inflammatory Myofibroblastic Tumor and Adenocarcinoma of the Colon: a Rare Entity
}

\author{
Chiara Cornacchia ${ }^{1}$, Mariangela Rutigliani ${ }^{2}$, Fiorenza Belli ${ }^{1}$, Nicola Romano ${ }^{3}$, Alberto Serventi ${ }^{4}$, Roberto Fiocca ${ }^{5}$, \\ Marco Filauro ${ }^{1}$
}

1) Department of Surgery,

Galliera Hospital, Genova;

2) Department of Pathology, Galliera Hospital, Genova;

3) Department of Radiology,

Galliera Hospital, Genova;

4) Department of Surgery,

Monsignor Galliano Hospital,

Acqui Terme (AL);

5) Department of Pathology,

San Martino Hospital, Genova, Italy

\section{Address for correspondence: \\ Nicola Romano \\ Department of Radiology, \\ Galliera Hospital, \\ Genoa, Italy. \\ romano.nicola@live.it}

Received: 23.01 .2020

Accepted: 18.05.2020

\begin{abstract}
This report presents the case of an 83-year old man with a collision tumor consisting of an inflammatory myofibroblastic tumor (IMT) and adenocarcinoma of the left colon. As the clinical and radiologic features of IMT are non-specific, only the accurate histopathological examination from the left hemicolectomy specimen was diagnostic. Although the prognosis of a colorectal IMT seemed more favorable than in other sites, four months after surgery the patient developed a tumor relapse. Therefore, malignant behavior of IMT could not be totally excluded. Recent studies have demonstrated that a chromosomal rearrangement involving 2p23, the site of the anaplastic lymphoma kinase $(A L K)$ gene, is present in a subset of these tumors. In our patient, tumor cells did not present ALK-1 perinuclear positivity and it could have indicated a less favorable prognosis. The collision of these different entities is extremely rare and this is the first case reported in literature. Further cases of collision tumors with clinical information including their treatment and prognosis are needed.
\end{abstract}

Key words: collision tumor - adenocarcinoma - inflammatory myofibroblastic tumor - colorectal surgery.

Abbreviations: ALK: Anaplastic lymphoma kinase gene; CT: computed tomography; IMT: inflammatory myofibroblastic tumor.

\section{INTRODUCTION}

In literature, collision tumors which have occurred in the colon consist of a combination of adenoma or adenocarcinoma with lymphoma, neuroendocrine tumors or leiomyosarcoma [1-3]. Here, we report the first case of a collision tumor composed of colon adenocarcinoma and an inflammatory myofibroblastic tumor (IMT). The colorectal IMT could not be distinguished clinically and radiologically. The diagnosis was based on detailed histology and immunohistochemistry (alfasmooth-muscle actin, vimentin, CD117, CD34, desmin, cytokeratin 20 and S-100). Although the behavior of IMT is generally benign, this type of tumor has a propensity for local recurrence; it rarely metastasizes.
The present report emphasizes the importance of detailed histopathologic and immunohistochemical analyses and clinical history along with a thoughtful discussion between the clinician and the pathologist in better defining a diagnosis.

\section{CASE REPORT}

An 83-year old Caucasian man presented to our emergency department after two days of diffuse abdominal pain, more acute in the lower left side. Physical examination revealed tenderness of the lower abdomen and a palpable mass in the lower left side. Laboratory results presented no remarkable abnormalities. A contrast-enhanced computed tomography (CT) scan demonstrated the presence of a nodular and polycyclic lesion of the left colon, with infiltration of submucosa, associated with increased attenuation of perivisceral fat. A colonoscopy revealed an ulcerative tumor in the descending colon and diverticulosis of the left colon. The biopsy specimen from the mucosal lesion confirmed the diagnosis of adenocarcinoma. A left hemicolectomy was performed, and the regional lymph nodes were removed. Intraoperatively, the descending colon was found edematous with a firm, cerebriform mass. The gross examination of the specimen showed a protruded, fullthickness tumor arising from the mesentery, $13 \mathrm{~cm}$ in diameter, white-grayish, with hard consistency, without necrosis or 
hemorrhages and a $3 \mathrm{~cm}$ full-thickness lesion arising from the mucosa with infiltration of the perivisceral adipose tissue (Fig. 1A). Microscopic examination revealed a collision tumor composed of two histologically distinct neoplasms at the same location: invasive tubular-type adenocarcinoma and IMT of the left colon (Fig. 1B). The diagnosis of IMT was documented by the presence of spindle cells with fascicular arrangement and mononuclear leukocytes (Fig. 1C). Immunohistochemically, the elongated tumor cells presented positivity for vimentin and focal positivity for alpha-smooth muscle actin (SMA) (clone 1A4, 1/100, Cell Marque). The cells did not express desmin (clone DE-R-11, 1/100, Ventana). Also CD34 (clone QBEnd/10 1/100, Ventana), CD117 (clone SP26, 1/100, Spring Bioscience), cytokeratin 20 (clone SP33, 1/100, Ventana) and DOG-1 (clone SP 31, 1/100, Cell Marque) antibodies were negative. Ki-67 (clone 30-9, 1/100, Ventana) positivity index was approximately $30 \%$ (Fig. 1D). The tumor cells did not present ALK-1 perinuclear positivity by immunohistochemistry and fluorescence in situ hybridization. The second tumor was an infiltrating adenocarcinoma moderately differentiated (Cytokeratin 20 positivity) that penetrated the subserosa without lymphatic and venous invasion (pT3G2N0). All lymphnodes isolated were negative for the presence of tumor cells. The patient developed an anastomotic leakage five days after surgery, confirmed by CT imaging. A laparotomy with colostomy was performed. The patient was discharged on the thirty-eighth post-operative day. The multidisciplinary team did not recommend postoperative adjuvant therapy. Four months after surgery, the patient presented to emergency department complaining of abdominal pain and fever. The CT examination demonstrated multiple and confluent masses with central areas of colliquative necrosis, that almost completely occupied the central and left abdomen: the inferior pole of the left kidney and the ipsilateral ureter, the small bowel loops and the anterior abdominal wall were infiltrated. No surgical measures were applied. A few days after the hospital admission the patient died of septic shock.

\section{DISCUSSION}

Collision tumors are rare clinical entities wherein two histologically distinct tumor types occur at the same anatomic site. Collision of the two malignancies can occur between tumors originating in the same organ or between metastases from other sites [4]. In literature, collision tumors of the colon consist of a combination of adenoma or adenocarcinoma with lymphoma, neuroendocrine tumors or leiomyosarcoma [1-3]. To the best of our knowledge, this is the first case of a colon collision tumor composed of adenocarcinoma and IMT.

Inflammatory myofibroblastic tumors are in themselves rare and poorly studied. The first description of IMT as a primary lung tumor dates back to 1937 [5]. Gastrointestinal IMTs are uncommon; in the colorectal segment, the first case of IMT was described by Coffin et al. in 1995 [6]. Colorectal IMTs can arise anywhere; the descending colon and sigmoid colon seem to be the most frequently involved sites [7]. They
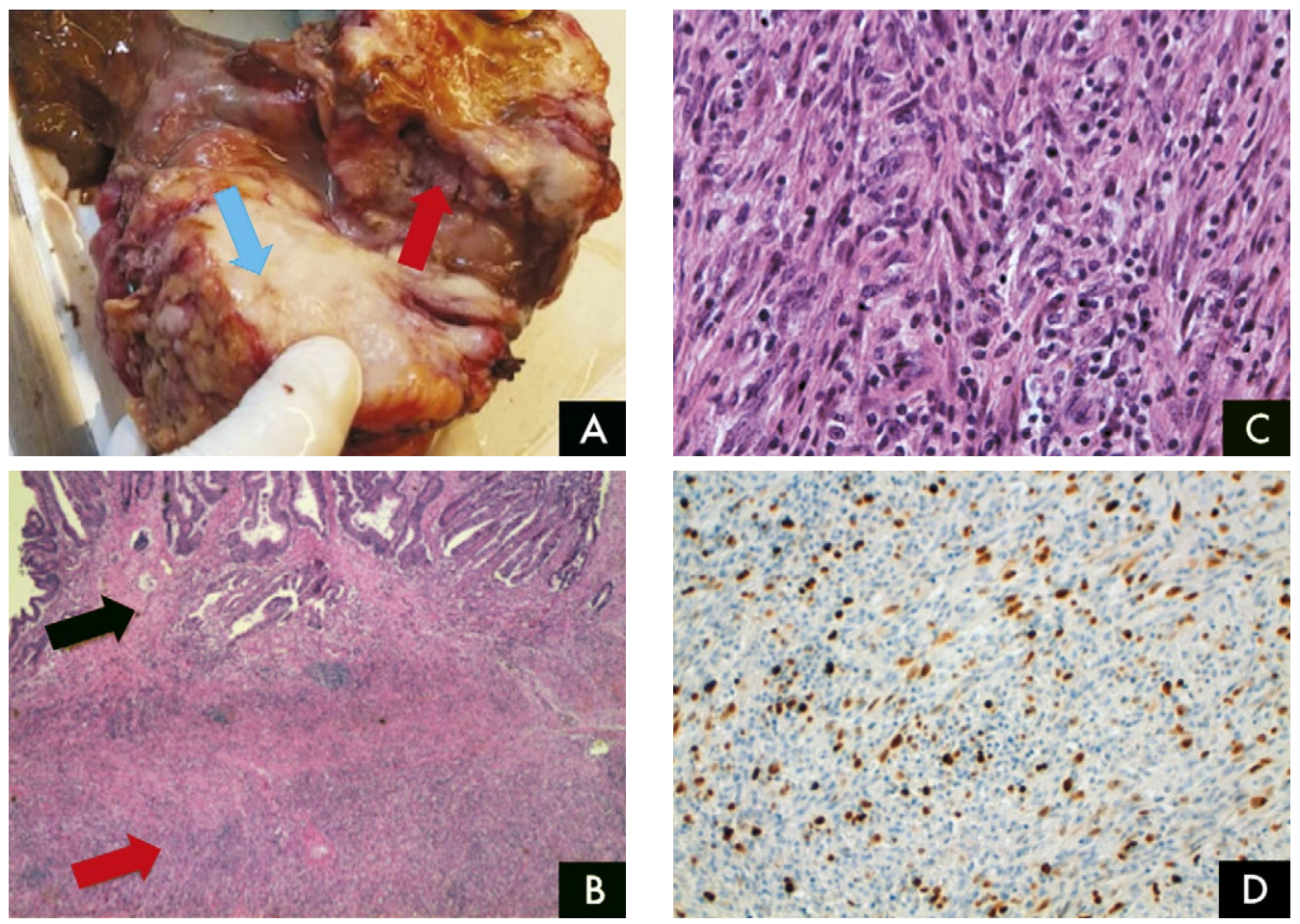

Fig. 1. Fig. 1.A): Macroscopically, the resected left colon revealing an ab-extrinsic intramural node of 13 $\mathrm{cm}$ (blue arrow) and an additional $3 \mathrm{~cm}$ full-thickness lesion arising from the mucosa with infiltration of the perivisceral adipose tissue (red arrow). B): Collision tumors: Invasive tubular-type adenocarcinoma (black arrow) and inflammatory myofibroblastic tumor of the left colon (red arrow) (hematoxylin-eosin staining, 4x magnification). C): Microscopically, the inflammatory myofibroblastic tumor was composed of spindle cells admixed with inflammatory cells (hematoxylin-eosin staining, 40x magnification). D): Expression of Ki67 staining (30\%) in inflammatory myofibroblastic tumor (KI-67 Mib1, 20 x magnification). 
may affect individuals of any age but have a predilection for children and young adults [8]. Patients may present nonspecific symptoms, such as anemia, abdominal pain and loss of weight, as in our case. The clinical features of IMT are the same as colorectal carcinoma and endoscopic examinations do not reveal any relevant differences between the two entities. Contrast-enhanced CT is the most useful imaging technique in the detection of a tumoral mass. Inflammatory myofibroblastic tumors usually appear hypodense, heterogeneous and welldeliniated; however, the CT findings are not univocal and the differential diagnosis includes: gastrointestinal stromal tumors (GIST), inflammatory fibroid polyp, smooth muscle neoplasm, peripheral nerve sheath tumour, fibromatosis, sarcoma, lymphoma and adenocarcinomas [9]. Therefore, CT is not sufficient to diagnose pre-operatively IMT, and a biopsy or surgery are required. The recurrence rate of gastrointestinal IMT is $18 \%$ to $40 \%$ [10]. Microscopically, IMT shows a similarity to GIST. An inflammatory myofibroblastic tumor is composed of the proliferation of spindled to epithelioid myofibroblasts with admixed inflammatory cells, predominantly lymphocytes. In the gastrointestinal tract, the interstitial cells of Cajal, which express CD34, could be considered pluripotent stromal cells. Gurzu et al. [11] hypothesized that the Cajal cells could be the precursor either for GIST, which keeps the expression of CD117 and CD34, or for IMT, which loses the positivity for CD34 and CD117 [11]. Although the prognosis of a colorectal IMT seems more favorable than in other sites, without reported recurrences between 10 months and 4.5 years after surgery [12, 13], malignant behavior cannot be totally excluded as in our casereport. Recent studies have demonstrated that a chromosomal rearrangement involving $2 \mathrm{p} 23$, the site of the $A L K$ gene, is present in a subset of these tumors [14]. It seems that IMTs with nuclear membrane or perinuclear ALK immunoreactivity have a low risk of relapse and they can also respond to treatment with a tyrosine-kinase inhibitor, crizotinib [11]. In our patient, tumor cells did not present ALK-1 perinuclear positivity and it can indicate a less favorable prognosis. The role of radiotherapy in IMT is unknown, although it may have potential benefits, particularly in unresectable cases. The treatment of choice for colorectal IMT remains surgical excision.

\section{CONCLUSION}

Our case of a colonic collision tumor composed of IMT and adenocarcinoma is unique, without literature references regarding the prognosis and the treatment. Further cases of collision tumor with clinical information, treatment options and prognosis are required.

Conflicts of interest: None to declare.
Authors' contribution: C.C and M.R. drafted the manuscript. All authors critically revised the manuscript, approved the final version to be published, and agree to be accountable for all aspects of the work.

\section{REFERENCES}

1. Kus T, Aktas G, Kalender ME, et al. Collision tumor consisting of primary follicular lymphoma and adenocarcinoma in the cecum: A case report and literature review. Oncol Lett 2016;11:2801-2805. doi:10.3892/ ol.2016.4310

2. Meeks MW, Grace S, Chen Y, et al. Synchronous Quadruple PrimaryNeoplasms: Colon Adenocarcinoma, Collision Tumor of Neuroendocrine Tumor and Schwann CellHamartoma and Sessile SerratedAdenoma of the Appendix. Anticancer Res 2016;36:4307-4311.

3. Kim VM, Goicochea L, Fang SH. Case Report: Collision Tumour of Colon Leiomyosarcoma and Adenocarcinoma. Clin Diagn Res 2016;10:PD03-PD04. doi:10.7860/JCDR/2016/16949.7956

4. Murthaiah P, Truskinovsky AM, Shah S, Dudek AZ. Collision tumor versus multiphenotypic differentiation: a case of carcinoma with features of colonic and lung primary tumors. Anticancer Res 2009;29:1495-1497.

5. Kim EY, Lee IK, Lee YS, et al. Inflammatory myofibroblastic tumor in colon. J Korean Surg Soc 2012;82:45-49. doi:10.4174/jkss.2012.82.1.45

6. Coffin CM, Watterson J, Priest JR, Dehner LP. Extrapulmonary inflammatory myofibroblastic tumor (inflammatory pseudotumor). A clinicopathologic and immunohistochemical study of 84 cases. Am J Surg Pathol 1995;19:859-872. doi:10.1097/00000478-199508000-00001

7. Aalbers AG, De Wilt JH, Zondervan PE, Ijzermans JN. A colonderived inflammatory pseudotumor. Dig Dis Sci 1999;44:578-581. doi:10.1023/a:1026665609461

8. Sanders BM, West KW, Gingalewski C, Engum S, Davis M, Grosfeld JL. Inflammatory pseudotumor of the alimentary tract: Clinical and surgical experience. J Pediatr Surg 2001;36:169-173. doi:10.1053/ jpsu.2001.20045

9. Cantera JE, Alfaro MP, Rafart DC, et al. Inflammatory myofibroblastictumours: a pictorial review. Insights Imaging 2015;6:8596. doi:10.1007/s13244-014-0370-0

10. Patnana M, Sevrukov AB, Elsayes KM, Viswanathan C, Lubner M, Menias CO. Inflammatory pseudotumor: the great mimicker. AJR Am J Roentgenol 2012;198:W217-W227. doi:10.2214/AJR.11.7288

11. Gurzu S, Bara T, Jung I. Inflammatory myofibroblastic tumor of the colon. J Clin Oncol 2013;31:e155-e158. doi:10.1200/JCO.2012.42.8961

12. Salgado-Sánchez E, Flores-Flores J, Pérez-Toriz MU, Pérez-Cruz R, Salgado-Sánchez J. Myofibroblast tumor. Rev Gastroenterol Mex 2003;68:219-221.

13. Zhou X, Luo C, Lv S, Gan M. Inflammatory myofibroblastic tumor of the rectum in a 13-month-old girl: a case report. J Pediatr Surg 2011;46:E1E4. doi:10.1016/j.jpedsurg.2011.03.011

14. Kovach SJ, Fisher AC, Katzman PJ, et al. Inflammatory myofibroblastic tumors. J Surg Oncol 2006;94:385-391. doi:10.1002/jso.20516 